ThP.IV-02 HIF Symposium, San Diego, March 13-17, 2000

\title{
Progress Toward Source-to-Target Simulation ${ }^{1}$
}

\author{
D. P. Grote, A. Friedman, G. D. Craig, W. M. Sharp \\ Lawrence Livermore National Laboratory \\ I. Haber \\ Naval Research Laboratory
}

\begin{abstract}
Source-to-target simulation of an accelerator provides a thorough check on the consistency of the design as well as a detailed understanding of the beam behavior. Issues such as envelope mismatch and emittance growth can be examined in a self-consistent manner, including the details of accelerator transitions, long-term transport, and longitudinal compression. The large range in scales, from centimeter-scale transverse beam size and applied field scale-length, to meter-scale beam length, to kilometer-scale accelerator length, poses a significant computational challenge. The ever-increasing computational power that is becoming available through massively parallel computers is making such simulation realizable. This paper discusses the progress toward sourceto-target simulation using the WARP particle-in-cell code. Representative examples are shown, including 3-D, long-term transport simulations of Integrated Research Experiment (IRE) scale accelerators.
\end{abstract}

Corresponding author: Dr. D. P. Grote

LBNL, B47-112

1 Cyclotron Rd.

Berkeley, CA 94720

Fax: 510-486-5392

Phone: 510-495-2961

Email: dpgrote@1bl.gov

PACS codes:

29.27-a Beams in particle accelerators

29.27.Eg Beam handling; beam transport

52.65.Rr Particle-in-cell method

Keywords:

Accelerator, Fusion, Heavy-ion, Induction, Simulation, Particle-in-cell, Plasma, Beam

1. This work was performed under the auspices of the U.S. Department of Energy by University of California Lawrence Livermore National Laboratory under contract No. W-7405-Eng-48, and by Naval Research Laboratory, and Lawrence Berkeley National Laboratory under contracts DE-AI0293ER40799, DE-AI02-94ER54232, and DE-AC03-765F00098. 


\section{Introduction}

A fundamental issue in heavy-ion-driven inertial confinement fusion energy is focusing the ion beam down onto the target. Whether or not this can be achieved depends on nearly all aspects of the system, from the required spot size (the target design), the chamber conditions, the final focus standoff, and the state of the beam, to name a few. The state of the beam depends on such issues as the design of the accelerator, the error tolerances, the behavior of waves on the beam, the ion source, etcetera. A complete understanding of the state of the beam at the target requires that the issues be examined in an integrated manner. Computer simulation is an ideal tool for this integrated examination. In this paper is discussed some of the progress toward complete source-totarget simulation of the beam.

The goal of the source-to-target simulation is to provide a fully consistent, fully integrated model of the beam, tracking the same particles from creation in the ion source, through acceleration and transport, through final compression and focus, and to the target. Tracking the same particles through the system removes any approximations that would be made by re-initializing the beam at the start of each section using low-order moments from the end of the previous section. The full detail of the dynamics is carried over from section to section.

The principal code being developed for the source-to-target simulation is the WARP[1] code. WARP combines the particle-in-cell (PIC) method from plasma physics with a detailed description of the accelerator lattice. The PIC method captures the beam dynamics, including the selffield, in a self-consistent manner. The model can either be three-dimensional (WARP3d), or two dimensional, axisymmetric (WARPrz) or transverse-slice (WARPxy). The description of the lattice includes accelerator elements having idealized axially uniform, hard-edged fields, as well as generalized elements where the applied field is specified either via axially varying multipole com- 
ponents or via data on a three-dimensional mesh. Electric elements can also be included self-consistently by including the geometry of the element as a boundary condition of the solution of Poisson's equation for the self-field. Additionally, there is an envelope solver which is primarily used to calculate the matched beam parameters in order to load the particles for the PIC models, when not injecting from the source.

Much work has been carried out in simulating various aspects of an accelerator. In these proceedings are papers describing simulation of the ion source and injector system[2,3], final focus[4], and propagation in the fusion chamber[5,6]. In this paper is discussed simulations of the acceleration section, after injection and before the final compression. This section is likely the longest and the most complex.

An additional aspect of the source-to-target work is general code development. This work is described elsewhere. Two major areas are development of improved methods of simulation, such as improved algorithms for particle codes and Vlasov methods[7,8], and development of models for the high-energy end of the accelerator, such as Darwin field models for magnetostatic and inductive fields[8], and multiple beam models[9].

\section{Transverse slice simulations}

Considerable understanding of the beam behavior in the accelerating section can be obtained using the transverse slice model. The model includes the two transverse position coordinates and all three velocity coordinates. A major use of the slice model is characterization of the design tolerances and allowable errors.

For a place to begin the simulations, a preliminary design of an IRE[10] scale accelerator was

drafted. The design is not necessarily optimized and is only representative. Some of the parameters and scaling relations are given in Table 1. The design consists of three sections. In the first, 
electric transverse focusing is used and the beam length (in meters) is held constant. In the second section, which begins the magnetic transverse focusing, the beam is compressed longitudinally. In the final section, the beam length is held constant and the accelerating gradient is at its maximum value. Using the continuous scaling relations and the transition points, a discrete lattice is laid out, ignoring the discontinuities in the velocity tilt. For the slice simulations, no accelerating waveforms need to be generated. Considerable work has been carried out characterizing a number of machine and beam errors in the IRE design.[11]

One issue is the alignment tolerance of the focusing quadrupoles. Any transverse offsets will kick the beam off-axis, possibly (in combination with non-linear applied fields and image forces) causing emittance growth and in the worst case causing some of the beam to be lost by striking the surrounding walls. In earlier work[11], the magnitudes of random quadrupole offsets were set so that, over the course of the simulation, little of the beam was lost to the walls. While it was known that the magnitudes of the errors were smaller than what could currently be achieved in experiment, the centroid displacements of the beam were representative of what would be expected in experiments which include steering.

Simulations using experimentally achievable quadrupole displacement errors along with beam steering have now been carried out. The offset errors in the electric quadrupoles were taken to have an RMS of 100 microns, based on estimated machining limits of the quadrupole structure. The errors in the magnetic quadrupoles are larger, 250 microns, obtained from estimates of the errors in magnet designs. The error distribution is Gaussian.

The steering used in the simulations is idealized. At the steering station, the centroid position of the beam is subtracted out and a small random error component added back in. To add this error component, which accounts for the steering errors (both in the sensing of the beam location and in 
the fields correcting it), the beam centroid is placed randomly within a circle of radius 200 microns about the axis. Any transverse velocity of the centroid is completely removed.

A series of simulations was carried out with different frequencies of steering, ranging from the best-case every half lattice period (HLP) up to once every forty HLP's. The results are summarized in Table 2. For each of the steering frequencies, six runs were done with differing sets of random errors. The pipe radius is $3.48 \mathrm{~cm}$. The numerical parameters are: $64 \times 64$ field grid, 40,000 simulation particles, 60 steps per half-lattice-period. The quadrupole fields include fringes with the pseudo-octopole term and the lowest order longitudinal component.

In Figure 1 are shown some of the results. In all of the cases the emittance growth is within acceptable limits. These simulations provide guidelines for how steering will be done in a large scale accelerator. They indicate that the beam can be steered back onto the axis fairly infrequently without causing significant degradation. Note that the RMS measure of beam offset is the more important, since the maximum can be anomalously large in the unlikely case that the errors resonate with the betatron motion of the centroid. More simulations are required for a thorough examination of the offsets in combination with other errors and non-linear fields.

\section{Three-dimensional Simulations}

There are a good number of issues which cannot be fully examined with the transverse slice model and so require a three-dimensional model. For example, the injection of the beam including transients and the initial "catching" by shaped accelerating waveforms, preventing head and tail expansion; head-to-tail variations in the mismatch and centroid offsets; and errors in the accelerating and ear waveforms.

For a three-dimensional simulation, a complete lattice design is required, including the accelerating waveforms. For the simple case of "load and fire", the voltage gradient on all of the gaps is 
the same at any time - all of the waveforms can be generated directly from the continuous scaling rules. With "load and fire", the beam drifts out of the injector until it is completely in the accelerator and then all of the gaps are switched on to accelerate the beam as a whole - the beam maintains a constant length. Figure 2 shows example waveforms in the first section of a model IRE design.

Using the generated waveforms and the associated lattice, three-dimensional simulations were carried out. The long and thin beams require significant computation resources. The numerical parameters used are: grid dimensions 32×32x2048 for one transverse quadrant, 2,000,000 particles, and 10,000 time steps. The clock time for the run on 128 processors of the NERSC[12] T3E900 was 1.1 hours. This simulation was only over the first section of the IRE design, with ideal electric quadrupoles (with no offsets). The beam travels just over 43 meters, or 168 HLP's.

As an aside, it is important to note that good parallel efficiency has been obtained for up to several hundred processors. With more processors, the efficiency begins to drop because of the global nature of the required Poisson solve for the beam self-fields. Improved techniques are being examined. Because of their large scale, efficient use of state-of-the-art parallel computers is essential for the source-to-target simulations to be possible.

The simulation shows a fairly quiescent beam. Essentially no increase is seen in the transverse normalized emittance (it varies by a few percent). The level of mismatch in the head and tail is small, roughly $5 \%$. The difference in the transverse size in the head and tail is consistent with the velocity tilt, $30 \%$. The global (over the full beam) RMS axial velocity spread $(\Delta \beta / \beta)$ is initially $0.3 \%$ and changes little. The overall spread is due to a combination of three effects: a thermal velocity spread which is initialized to just under $0.3 \%$ (and equal to the transverse thermal velocity spread), and decreases inversely proportional to the increasing velocity; the variation of the 
axial velocity in the accelerating gaps which is initially $0.35 \%$ and increases with the velocity; and the variation of the head and tail velocities due to imperfect axially confining ear fields which initially is zero and increases to of order $1 \%$. With perfect ears, the estimated velocity spread would be just over $0.2 \%$. It is a coincidence that the three combined in this simulation to give a nearly constant spread. Over the course of the simulation, the local absolute velocity spread $(\Delta \beta)$ changes little.

Further work will begin including element errors, use realistic steering algorithms, examine other accelerating schemes (in addition to "load and fire"), and will include more of the accelerator past the electric focusing section. One alternate scheme to begin the acceleration of the beam can be called "fire and load". Here, the gaps are switched on as the beam enters the accelerator. The first several gaps are tailored to have a rapidly rising waveform to give the beam its initial velocity tilt, i.e. the tail of the beam is accelerated more initially so it keeps up with the head which has gone through more gaps. In Figure 3 is shown a sample set of waveforms for "fire and load". After the whole beam is in the accelerator, the waveforms of both schemes are similar. The "fire and load" scheme allows shortening of the accelerator length of order the initial beam length and removes constraints on the rise time of the gap voltages, at the expense of more complicated waveforms and a small but unavoidable longitudinal expansion of the beam.

\section{Conclusions}

A thorough validation of the beam dynamics in an IRE or driver accelerator requires integrated source-to-target simulation, tracking the same particles from birth to death. Significant simulations have been carried out of all parts of a large scale accelerator, including injection, acceleration, and final compression and focus. Much progress has been made toward full simulation of the main accelerator section, which is likely the most complex section. Both two-dimen- 
sional and three-dimensional simulations are being carried out. The next step will be connecting the simulations of the different sections.

\section{References}

[1] "Three-dimensional simulations of high current beams in induction accelerators with WARP3d", D. P. Grote, A. Friedman, I. Haber, S. Yu, Fus. Eng. \& Des., 32-33 (1996) 193-200. [2] "Multiple-beam ion guns for heavy-ion fusion", E. Henestroza, D. N. Beck, A. Faltens, D. P. Grote, J. W. Kwan, these proceedings.

[3] "The adiabatic matching section solution for the source injector", L. Ahle, D. P. Grote, E. Halaxa, these proceedings

[4] "Simulations of the LBNL scaled final focus experiment", M. J. L. de Hoon, E. P. Lee, these proceedings.

[5] "Improved modeling of chamber transport for heavy-ion fusion", W. M. Sharp, M. S. Armel, D. A. Callahan-Miller, A. B. Langdon, J.-L. Vay, these proceedings.

[6] "Ballistic neutralized chamber transport of intense heavy-ion beams", D. V. Rose, A. Friedman, B. V. Oliver, W. M. Sharp, D. R. Welch, these proceedings.

[7] "Simulation of heavy-ion beams with a semi-lagrangian Vlasov solver", E. Sonnendrucker, et. $a l$. , these proceedings.

[8] "Progress towards simulating heavy ion beams for inertial fusion energy based on 1) a Darwin model field solver, and 2) a semi-lagrangian Vlasov solver", E. Sonnendrucker, Proceedings of the 1999 Particle Accelerator Conference, p. 2758.

[9] "Beam simulation for IRE and driver - status and strategy", A. Friedman, et. al., these proceedings.

[10] "Planning for an integrated research experiment", J. J. Barnard, et. al., these proceedings.

[11] "Beam dynamics studies for heavy-ion fusion drivers", A. Friedman, et. al., Proceedings of the 1999 Particle Accelerator Conference, p. 1830.

[12] National Energy Research Scientific Computing Center, at Lawrence Berkeley Laboratory, Berkeley, California. 


\begin{tabular}{|c|c|c|c|c|c|c|c|}
\hline $\begin{array}{l}\text { Total Energy }=30 \mathrm{~kJ} \\
32 \text { Beams of } \mathrm{K}^{+} \text {ions } \\
\mathrm{A}=38.96 \mathrm{amu} \\
\sigma_{\mathrm{o}}=54^{\mathrm{O}} ; \sigma / \sigma_{\mathrm{o}} \sim 0.1 \\
\mathrm{a}_{\text {mean }}=1.8 \mathrm{~cm}\end{array}$ & $\begin{array}{l}\text { Energy } \\
(\mathrm{MeV})\end{array}$ & $\begin{array}{l}\text { Current } \\
\text { (Amps) }\end{array}$ & $\begin{array}{c}\text { Pulse } \\
\text { duration } \\
(\mu s)\end{array}$ & $\begin{array}{c}\text { Bunch } \\
\text { length } \\
(\mathrm{m})\end{array}$ & $\begin{array}{l}\text { HLP } \\
\text { length } \\
(\mathrm{m})\end{array}$ & Occupancy & $\begin{array}{c}\text { Accelerating } \\
\text { Gradient } \\
(\mathrm{MV} / \mathrm{m})\end{array}$ \\
\hline Initial & 1.6 & 0.77 & 6.7 & 18.8 & 0.20 & 0.65 & 0.055 \\
\hline Electric Focusing & $\mathrm{V}$ & $\mathrm{v}^{1 / 2}$ & $\mathrm{v}^{-1 / 2}$ & $\mathrm{v}^{0}$ & $\mathrm{v}^{1 / 2}$ & $\mathrm{v}^{0}$ & $\mathrm{v}$ \\
\hline E-M transition & 9.4 & 1.9 & 2.8 & 18.8 & 0.50 & 0.33 & 0.32 \\
\hline \multirow[t]{2}{*}{ Compression } & $\mathrm{v}$ & $\mathrm{v}$ & $\mathrm{v}^{-1}$ & $\mathrm{v}^{-1 / 2}$ & $\mathrm{v}^{1 / 4}$ & $\mathrm{v}^{0}$ & $\mathrm{v}$ \\
\hline & 30. & 5.9 & 0.87 & 10.6 & 0.67 & 0.33 & 1.0 \\
\hline Constant accel & $\mathrm{v}$ & $\mathrm{v}^{1 / 2}$ & $\mathrm{v}^{-1 / 2}$ & $\mathrm{v}^{0}$ & $\mathrm{v}^{1 / 2}$ & $\mathrm{v}^{-1 / 2}$ & $\mathrm{v}^{0}$ \\
\hline Final & 200. & 15. & 0.34 & 10.6 & 1.7 & 0.13 & 1.0 \\
\hline
\end{tabular}

Table 1: The variation of lattice and beam parameters as functions of the beam mid-pulse energy, v. There are three sections: in the first electric focusing is used; in the second, magnetic focusing is used and the beam is compressed; and in the third, the beam is not compressed and the accelerating gradient is held constant at its maximum value. The numbers shown are for a specific model design and do not represent an optimal for final design. 


\begin{tabular}{|c|c|c|}
\hline $\begin{array}{c}\text { Steering } \\
\text { frequency } \\
\text { (HLP's })\end{array}$ & $\begin{array}{c}\text { RMS offset } \\
(\mathrm{mm})\end{array}$ & $\begin{array}{c}\text { Max offset } \\
(\mathrm{mm})\end{array}$ \\
\hline 1 & 0.11 & 0.62 \\
\hline 5 & 0.3 & 3.7 \\
\hline 10 & 0.8 & 4.6 \\
\hline 20 & 1.2 & 6.4 \\
\hline 40 & 1.5 & 8.0 \\
\hline
\end{tabular}

Table 2: Beam centroid RMS offsets and maxima for transverse slice simulations with realistic quadrupole offsets and ideal steering. The quadrupole offsets are $100 \mu$ for the electric quadrupoles and $250 \mu$ for the magnetic. The steering places the beam within $200 \mu$ of the axis and removes the centroid velocity. 
a)

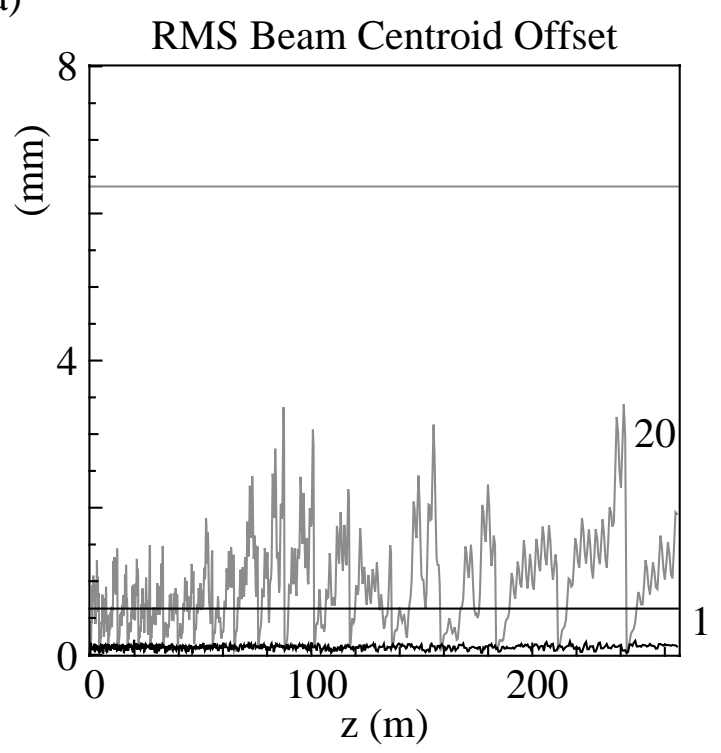

b)

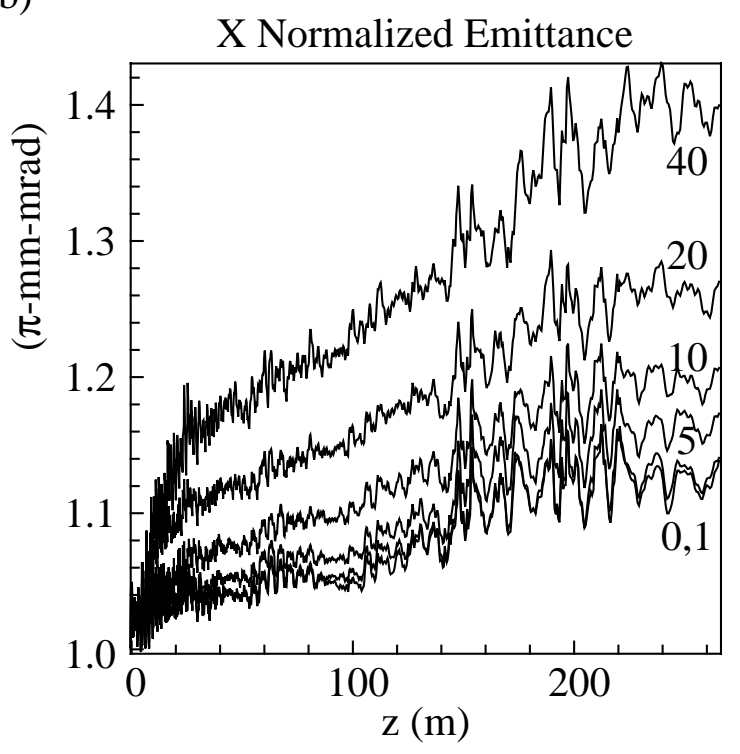

Figure 1: Results from transverse slice simulations with quadrupole offsets and ideal steering. a) The RMS beam centroid offset. The black is the ideal case of steering every HLP. The gray is steering every twenty HLP's. The two jagged curves show, for the two cases, the RMS over six simulations for each $\mathrm{z}$ location. The horizontal lines show the maximal value over all of the simulations. b) The $x$ normalized RMS edge emittance for the cases with no errors, and with errors and steering every $1,5,10,20$, and 40 HLP's. 


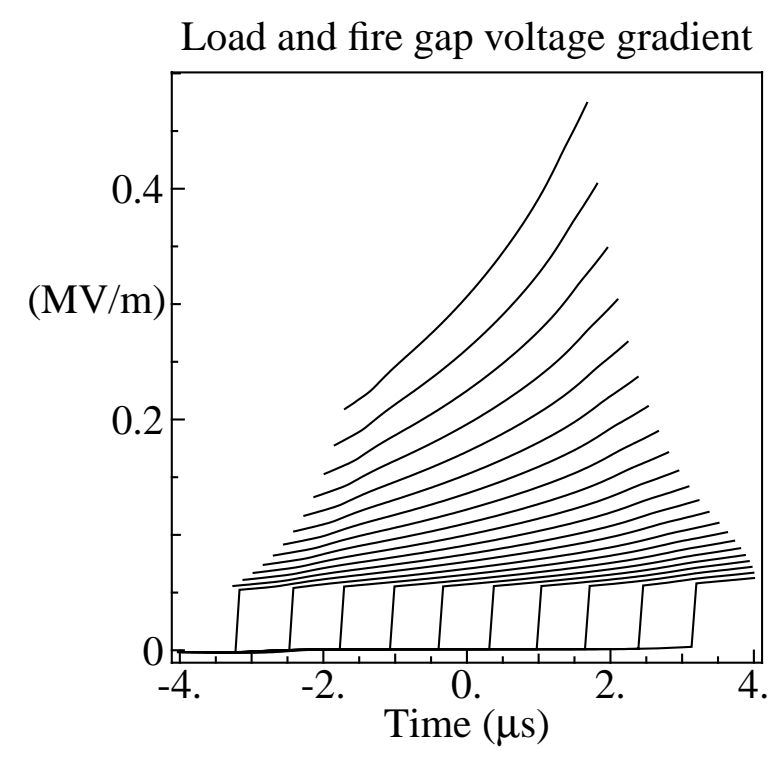

Figure 2: The gap voltage gradient for every tenth gap using the load and fire procedure, plotted relative to the time of arrival of the center of the beam at the gap. 


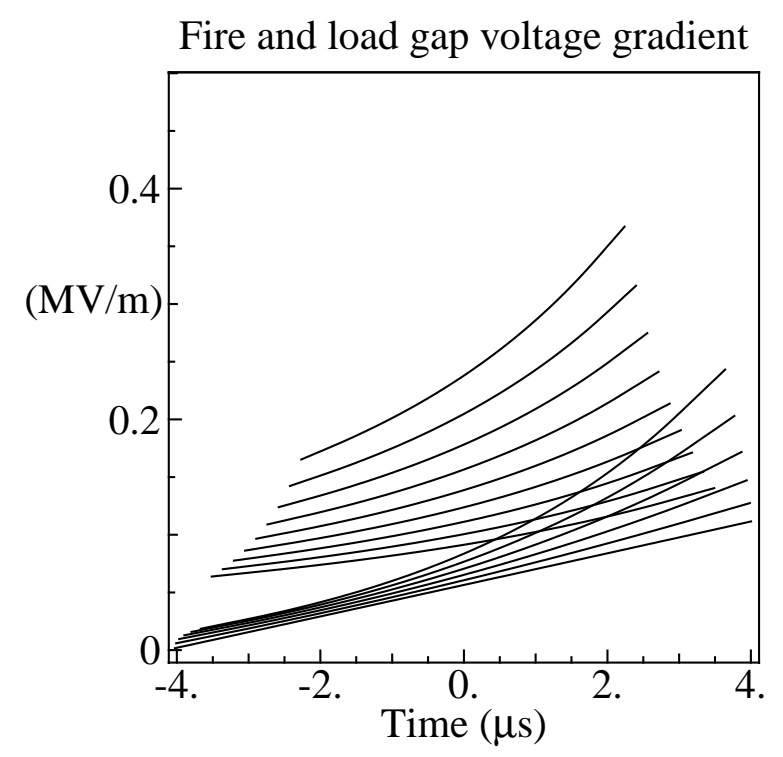

Figure 3: The gap voltage gradient for every tenth gap using the fire and load procedure, plotted relative to the time of arrival of the center of the beam at the gap. The first six of the curves shown were tailored to gradually increase the axial velocity tilt. 